\title{
THE WAVELET-BASED DENOISING OF IMAGES IN FIJI, WITH EXAMPLE APPLICATIONS IN STRUCTURED ILLUMINATION MICROSCOPY
}

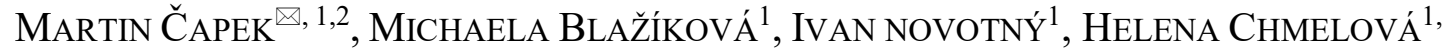 \\ DAVID SVOBODA ${ }^{3}$, BARBORA RADOCHOVÁ ${ }^{2}$, JIŘí JANÁČEK ${ }^{2}$, ONDREJ HORVÁTH ${ }^{1}$ \\ ${ }^{1}$ Institute of Molecular Genetics of the Czech Academy of Sciences, Light Microscopy Core Facility, Prague, \\ Vídeňská 1083, Prague 4, 14220 Czech Republic, ${ }^{2}$ Institute of Physiology of the Czech Academy of Sciences, \\ Laboratory of Biomathematics, Vídeňská 1083, Prague 4, 14220 Czech Republic, ${ }^{3}$ Masaryk University, Fac- \\ ulty of Informatics, Centre for Biomedical Image Analysis, Botanická 68a, Brno, 60200 Czech Republic \\ e-mail: martin.capek@img.cas.cz,michaela.blazikova@img.cas.cz, ivan.novotny@img.cas.cz, \\ helena.chmelova@img.cas.cz, svoboda@fi.muni.cz, barbora.radochova@fgu.cas.cz,jiri.janacek@fgu.cas.cz, \\ ondrej.horvath@img.cas.cz
}

(Received July 1, 2020; accepted December 8, 2020)

\begin{abstract}
Filtration of super-resolved microscopic images brings often troubles with removing undesired image parts like, e.g., noise, inhomogenous background and reconstruction artifacts. Standard filtration techniques, e.g., convolution- or Fourier transform-based methods are not always appropriate, since they may lower image resolution that was acquired by hi-tech and expensive microscopy systems. Thus, in this article it is proposed to filter such images using discrete wavelet transform (DWT). Newly developed Wavelet_Denoise plugin for free available Fiji software package demonstrates important possibilities of applying DWT to images: Decomposition of a filtered picture using various wavelet filters and levels of details with showing decomposed images and visualization of effects of back transformation of the picture with chosen level of suppression or denoising of wavelet coefficients. The Fiji framework allows, for example, using a plethora of various microscopic image formats for data opening, users can easily install the plugin through a menu command and the plugin supports processing 3D images in Z-stacks. The application of the plugin for removal of reconstruction artifacts and undesirable background in images acquired by super-resolved structured illumination microscopy is demonstrated as well.
\end{abstract}

Keywords: discrete wavelet transform, Fiji plugin, image filtration, structured illumination microscopy.

\section{INTRODUCTION}

The commonly used low-pass filtering of super-resolved microscopic images does not bring expected results. If we try to remove undesired image parts, e.g., noise, inhomogenous background, reconstruction artifacts, by these techniques, we lose resolution that was acquired by hi-tech and expensive microscopy systems.

These methods suppose that noise and artifacts are small image parts that can be safely removed by some kind of convolution filtration, e.g., replacing a pixel intensity by a mean intensity of this neighborhood, or by lowering the range of image frequencies in case of Fourier filtering. The convolution-based filters work well, e.g., for photographic images, but cause blur and may increase the size of structures, which is not welcome in super-resolved microscopic images. Thus, another technique of image filtration that is able to preserve the size of structures and original resolution of microscopic data as much as possible would be desired.
One of promising techniques applied in digital image processing is a discrete wavelet transform (DWT) (Gonzales and Woods, 2008). Discrete Fourier transform captures image frequencies. On the contrary, DWT captures both frequency and location information. In other words, DWT is able to distinguish, e.g., that one object in the picture is large and homogenous in intensity in comparison with other objects in the same picture that are subtle with sudden intensity changes. Due to these properties, DWT is known to be interesting for the filtration of images.

Previously, several available tools for wavelet transformation of images have been developed. For example, interesting web-based demos were published (Sage and Unser, 2003). Two approaches shown here demonstrate applying Daubechies and Spline based DWT to images, while another one does picture denoising by soft-thresholding in the wavelet domain (Rangarajan et al., 2002). 
Probably, the most efficient support for waveletbased processing of signals and images has been implemented in Matlab (mathworks.com) in its Wavelet Toolbox. Especially, Wavelet Analyzer tool is worth noting here. Other interesting tools aimed directly at denoising single images by orthonormal wavelet thresholding were presented in Matlab code (Luisier et al., 2007; Luisier and Blue, 2008). Matlab-based libraries of third parties for wavelet analysis are, for example, WavBox Software Library (toolsmiths.com) and WaveLab, (statweb.stanford.edu/ wavelab).

A standalone application for the study of the wavelet transform (Visual Wavelet-Lab) has been described (Roselló et al., 2014), as well as simple applications for DWT - 2D Fast Wavelet Transform Library in C++ (codeproject.com/Articles/20869/D-Fast-Wavelet-

Transform-Library-for-Image-Pro-ces) and Wavelets demo in Accord.NET framework (accord-framework.net/samples.html). Xlib library with DWT of images (imagej.net/Xlib\#Wavelets 2D), Fractional Wavelet Module (2D or 3D) plugin with filtering possibilities (Unser and Blue, 2000), denoising single images using Haar filters (imagej.nih.gov/ij/plugins/haar-wavelet-filter.html) are, for example, available in ImageJ (imagej.nih.gov).

Mostly, the above described approaches are mentioned as demos or relatively simple applications for DWT of images. The exceptions are tools in Matlab, which is, however, not a free software package and filtering of 2D/3D multichannel microscopic images of various formats is not straightforward. Other exception is Xlib library of ImageJ plugins (imagej.net/Xlib\#Wavelets_2D) that offers a tool for doing forward and backward DWT of images with various wavelet families, but without inherent filtering wavelet coefficients. Another exception is comprehensive Fractional Wavelet Module (2D or 3D) plugin (Unser and Blue, 2000) with various filter coefficients processing possibilities prior image synthesis, however, which does not offer the most common wavelet families used nowadays.

Thus, in our opinion, a practical, freely available tool that can interactively carry out DWT based filtering of large 2D/3D microscopic images using latest wavelet families is still missing.

Fiji is an open-source, freely available, image processing package (Fiji.sc). It is a distribution of ImageJ (imagej.nih.gov) bundling many plugins which facilitate scientific image analysis. Fiji is a very popular choice of a software for image processing and analysis in microscopic research community. Plugins for Fiji can be eas- ily developed in Java and by using open-source integrated development environments, like Eclipse (www.eclipse.org).

Therefore, we developed a Fiji plugin called Wavelet_Denoise. The plugin follows a project called WTUTOR2D that provides an educational tool for wavelet transform and its application in the field of digital image processing (cbia.fi.muni.cz/software/wtutor2d.html; Sečkáŕ, 2016). Since we decided to provide large and worldwide Fiji community with interesting possibilities of WTUTOR2D project, we adapted it to Fiji.

The resulting plugin demonstrates main possibilities of applying DWT in Fiji to images: Decomposition of an input picture to DWT using various wavelet filters and levels of details with decomposed image visualization; Demonstration of effects of back transformation of the picture with some level of suppression or denoising of wavelet coefficients of the decomposed image; Fiji is famous for its ability to read images using a plethora of various microscopic formats; Fiji users can easily install the plugin through a menu command; The plugin supports processing 3D images in Z-stacks; Decomposed images can be stored in 32-bit format for analysis in other software packages (e.g., Matlab) if required; Approximation and detailed coefficients of DWT can be suppressed individually; One-Slice-Preview for processing large 3D images is offered as well.

In this work, we demonstrate the application of the plugin and wavelet filtering for removal of reconstruction artifacts and undesirable background in images acquired by super-resolved structured illumination microscopy.

\section{MATERIALS AND METHODS}

\section{DISCRETE WAVELET TRANSFORM}

To make it easier to understand the following description of the plugin, we find it useful to describe the basics of DWT here. For simplicity, we demonstrate principles of DWT first using a 1D signal (Strang and Nguyen, 1997). 1D DWT is defined:

$$
\begin{gathered}
A\left(j_{0}, k\right)=\frac{1}{\sqrt{N}} \sum_{n=0}^{N-1} f(n) \varphi_{j_{0}, k}(n), \\
D(j, k)=\frac{1}{\sqrt{N}} \sum_{n=0}^{N-1} f(n) \psi_{j, k}(n) \text { for } j \geq j_{0},
\end{gathered}
$$

where $f(n)$ is a signal to be transformed and $A, D$ are approximation and detailed coefficients, respectively. Iterations are controlled by $j \in\left\{j_{0}, \ldots, J-1\right\}$, where $J=$ $\log _{2} N, N$ is the length of the signal and should be a 
power of 2 , and $k \in\left\{0,1, \ldots, 2^{j}-1\right\}$. Here, $\varphi$ is called the scaling function, which is defined as

$$
\varphi_{j, k}(x)=\sqrt{2^{j}} \varphi\left(2^{j} \frac{x}{N}-k\right),
$$

where $k$ governs position and $j$ width of the scaling function. $\psi$ is called the wavelength function and is defined as

$$
\psi_{j, k}(x)=\sqrt{2^{j}} \psi\left(2^{j} \frac{x}{N}-k\right) .
$$
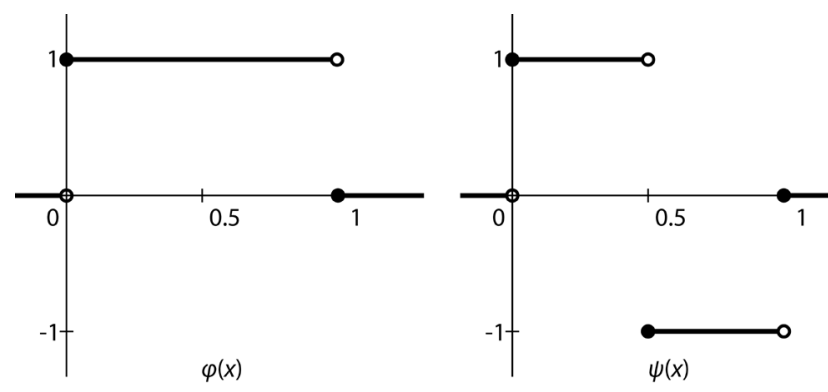

Fig. 1. Haar scaling $\varphi(x)$ and wavelet functions $\psi(x)$.

In case of Haar functions, which are the easiest functions used in practice (e.g., Gonzales and Woods, 2008), Fig. 1, the relation between the scaling and wavelength function can by simply described by

$$
\psi(x)=\varphi(2 x)-\varphi(2 x-1),
$$

see Fig. 2.

Back transformation for getting the original signal, i.e. synthesis, is then defined

$$
\begin{array}{rl}
f(n)=\frac{1}{\sqrt{N}} \sum_{k=0}^{2^{j_{0}}-1} & A\left(j_{0}, k\right) \varphi_{j_{0}, k}(n) \\
& +\frac{1}{\sqrt{N}} \sum_{j=j_{0}}^{J-1} \sum_{k=0}^{2^{j}-1} D(j, k) \psi_{j, k}(n) .
\end{array}
$$
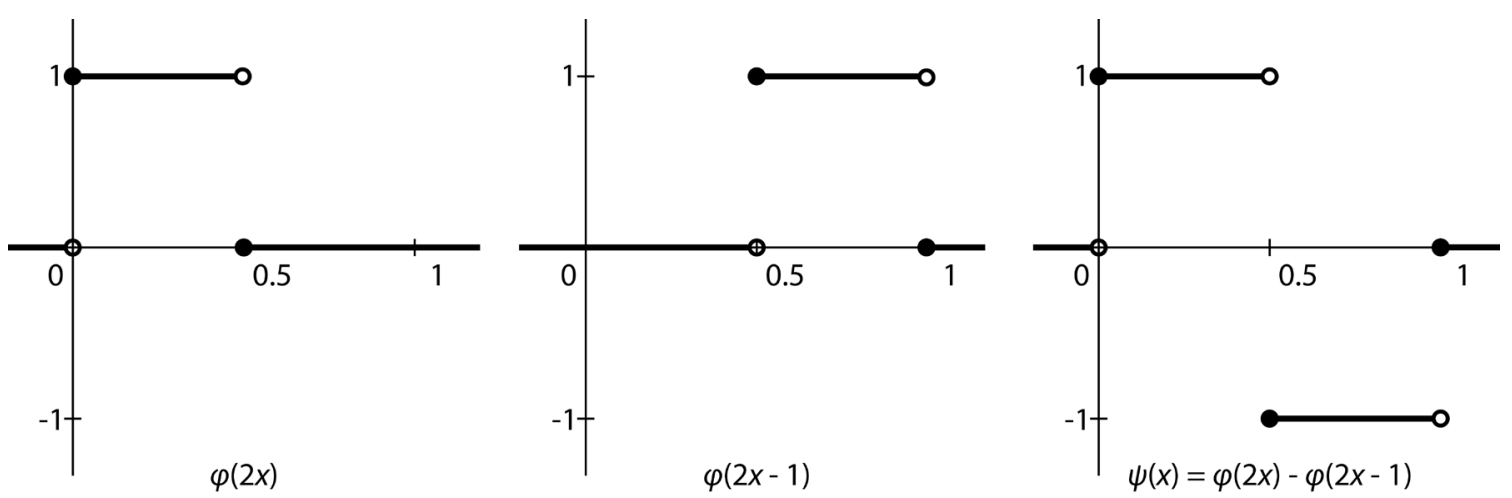

Fig. 2. The relation between the Haar scaling $\varphi(x)$ and wavelength $\psi(x)$ function. 


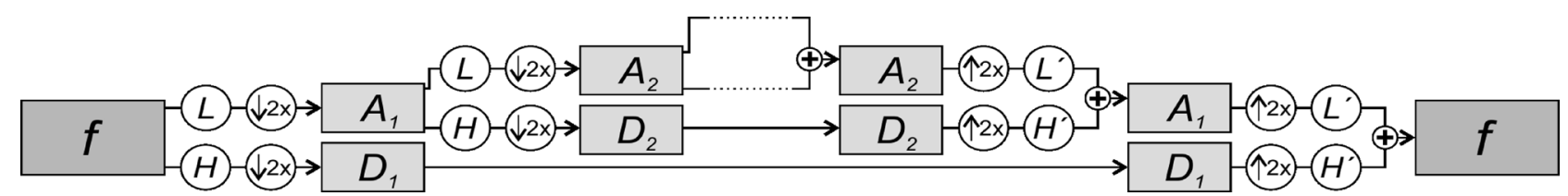

Fig. 4. Iterative analysis and subsequent signal synthesis. A and D after the first iteration are A1 and D1, after the second iteration $A 2$ and D2, etc.

Furthermore, after the first analysis of the signal $f$ we can also analyze the undersampled approximation coefficients $A$ and divide them again into the corresponding $A$ and $D$. These can also be downsampled, as they contain redundant data, and we can iteratively repeat the analysis, until the signal shrinks into one value. Then the original signal is reconstructed in a similar way, see Fig. 4. This technique is called subband coding.

The filters $L$ and $H$ for analysis and $L^{\prime}$ and $\mathrm{H}^{\prime}$ for signal synthesis, i.e. the scaling function $\varphi$ and the wavelet function $\psi$, must satisfy several conditions to ensure that the output signal matches the input. Socalled orthogonal or biorthogonal filters meet the required conditions. There are many such filters in the literature, such as Haar (Gonzales and Woods, 2008), shown above, Daubechies (Strang and Nguyen, 1997), or Meyer (Chui, 1992). In the way we have just described, i.e. by repeated filtration with suitable filters and resampling, we perform the forward and back wavelet transformations of the given signal.

The extension of 1D DWT to 2D is straightforward. The scaling and the wavelength functions are the following:

$$
\begin{aligned}
\varphi(x, y) & =\varphi(x) \varphi(y), \\
\psi^{H}(x, y) & =\psi(x) \varphi(y), \\
\psi^{V}(x, y) & =\varphi(x) \psi(y), \\
\psi^{D}(x, y) & =\psi(x) \psi(y) .
\end{aligned}
$$

$\varphi$ generates approximation coefficients and $\psi^{H}, \psi^{V}, \psi^{D}$ generate horizontal, vertical and diagonal detailed coefficients, respectively (Antonini et al., 1992; Gonzales and Woods, 2008). A 2D image of the size $N x N$ after the first iteration consists of four subbands, where approximation coefficients can be iterated further, with the restriction coming from the image size, i.e. $J$-times at most (Fig. 5). It should be also noted that thanks to the definitions of the scaling and wavelength functions (7)-(10), 2D DWT is easily separable and can, therefore, be done separately for each axis. Thus, in reality, 1D subband coding is performed line by line along the $x$ axis and then column by column along the $y$ axis in case of 2D DWT.

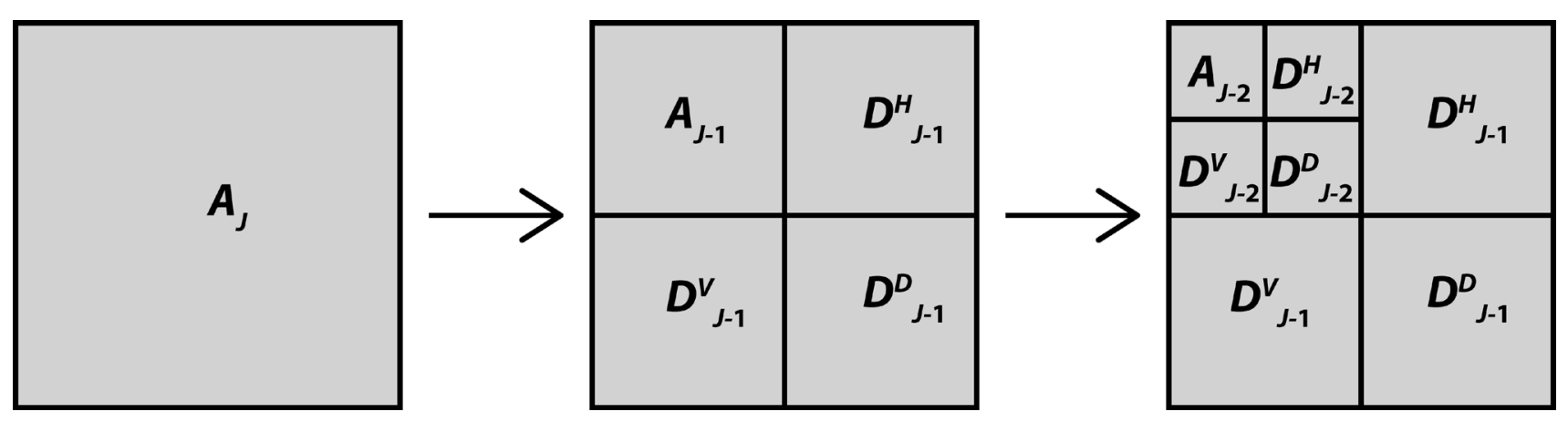

Fig. 5. Original image (left), the image after the first iteration of 2D-DWT (middle) and the image after the second iteration (right). Approximation after the first iteration is denoted $A_{J-1}$ and horizontal, vertical and diagonal details are denoted $D_{J-1}^{H}, D_{J-1}^{V}, D_{J-1}^{D}$. Approximations and details after multiple iterations are denoted by $J-2, J-3, \ldots, 1,0$. 

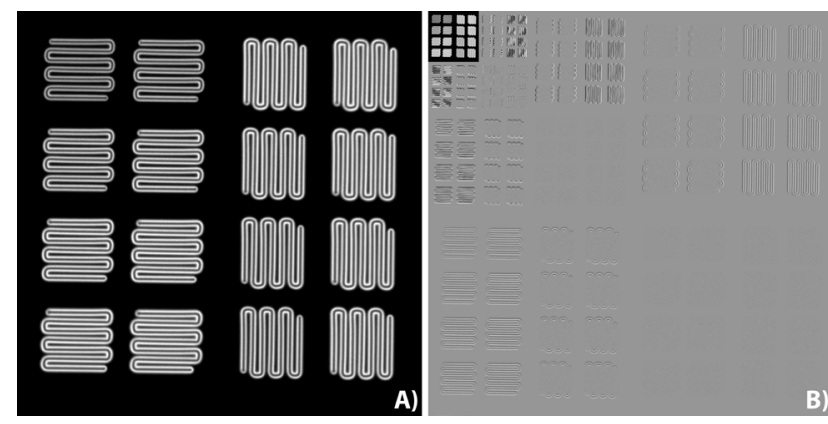

Fig. 6. Original 2D image (A) and its wavelet transformation (B) using Haar wavelet after three iterations, see text for detailed explanation. For better visualization, the transformed image values were linearly expanded.

An example of 2D DWT is given in Fig. 6 which shows the original image, structures acquired by a Leica SP8 confocal microscope from an Argolight confocal calibration slide (Royon and Converset, 2017), as well as the transformed image. When transforming a 2D signal, as explained above, we get approximation coefficients $A$ (i.e., the downsampled image in the upper left corner of the transformed image) and series of detailed horizontal coefficients $D^{H}$ (shown along the top horizontal edge of the image), detailed vertical coefficients $D^{V}$ (along the left vertical edge) and detailed diagonal coefficients $D^{D}$ (along the diagonal of the image).

When viewing the transformed image, it may be noted that the horizontal detailed coefficients capture the intensity changes within the columns of the image, i.e. vertical structures, the vertical detailed coefficients within the rows, i.e. horizontal structures, and the diagonal within the diagonals. The diagonal structures are practically not present in Fig. 6. Moreover, with greater submerging we observe larger structures and coarser features of the image.

\section{STRUCTURED ILLUMINATION MICROS- COPY (SIM)}

SIM is a super-resolution fluorescence light microscopy technique that breaks Abbé diffraction limit (Born and Wolf, 1997) and doubles resolution of standard fluorescence microscopes to about $100 \mathrm{~nm}$ (Gustafsson, 2000). SIM uses sinusoidal illumination grid pattern to create moiré that carries information about details in specimens. For obtaining the image it is necessary to acquire usually fifteen pictures per one optical layer using three rotations and five shifts of the grid. The super-resolved image is reconstructed with the help of Fourier spectra analysis from the acquired pictures (Gustafsson et al., 2008).
SIM is burdened by possible artifacts (Demerle et al., 2017). Haloing is doubling structures in the reconstructed image. This is caused either by refractive index mismatch in the system of specimen, immersion oil and objective, or by photobleaching. It can be avoided by careful preparation of specimens, optimizing the refractive index of the immersion oil and avoiding bleaching across the specimen.

Another artifact observed in SIM is a regular, hexagonal pattern referred to as "honeycomb" (Demerle et al., 2017). It is common in pictures of specimens with low signal to noise ratio (SNR). This artifact is pronounced especially by masking the SIM pattern by high level of background and/or by using samples with diffuse labeling. This can be cured by visualizing samples that have discrete structures, i.e. that do not show diffuse labeling, and by increasing SNR without photobleaching.

The quality of reconstructed pictures from SIM with regard to the artifacts can be also influenced by using appropriate parameters for SIM reconstruction, especially a function apodizing the frequency spectrum and the Wiener filter constant (Demerle et al., 2017). This constant can be determined either empirically or, e.g., using the Wiener setting recommendation in Modulation Contrast tool in SIMcheck plugin developed for Fiji (Ball et al., 2015).

There are several possible functions that can be used for apodizing the frequency spectrum. These are not always present in reconstruction software distributed by vendors of commercial structured illumination microscopes. However, one can find them, for example, in SIMToolbox package (Kř́ržek et al., 2016) that is distributed for free (integrated apodization functions: a standard incoherent model, cosine bell, Butterworth, triangle and Lukosz bound (Righolt et al., 2013)).

The apodization functions can lower noise in images, however, their stronger application reduce image resolution. Increasing the value of the Wiener filter constant decreases reconstruction artifacts, but this process lowers image resolution as well (Righolt et al., 2013). Thus, one should be careful with their manipulations.

Even with careful sample preparation, acquisition and reconstruction, it may still happen that artifacts appear in the SIM data. Here comes the use of filtration as a post-processing step. We propose to apply our Wavelet_Denoise plugin for Fiji, which is suitable for denoising and filtering image data. 


\section{WAVELET DENOISE FIJI PLUGIN}

When one runs the plugin in Fiji, the following dialogs and up to four pictures appear (Fig. 7), according to options chosen in the dialog. It should be noted that the plugin requires $2 \mathrm{D}$ image size dimensions to be powers of two, otherwise, the plugin announces a warning message and stops. If the condition is not met, it is possible to crop, scale, etc. the images directly in Fiji by using appropriate procedures. The plugin works both with $2 \mathrm{D}$ images and with 3D stacks (Fig. 7C shows the stacked original data) and processes the images in the wavelet domain.

The plugin runs in three steps. Firstly, it computes the forward DWT (analysis, decomposition; Fig. 7E).
Secondly, it does an operation in the wavelet domain using a chosen wavelet filter and the level of details ( Levels of Detail 1, Daubechies 4, Suppress AC 85\%, Suppress $D C 10 \%$ applied in the main dialog, Fig. 7B). Thirdly, it computes the inverse DWT from processed wavelet coefficients (synthesis, reconstruction, the result of filtration/denoising; Fig. 7D).

The dialog is modeless and accepts adjustments of the original image prior DWT processing in the plugin. Together with the main dialog and the pictures, the Synchronize Windows dialog (Fig. 7G) appears that gives possibility to change image zoom, slice position, etc. easily in all data windows at once.

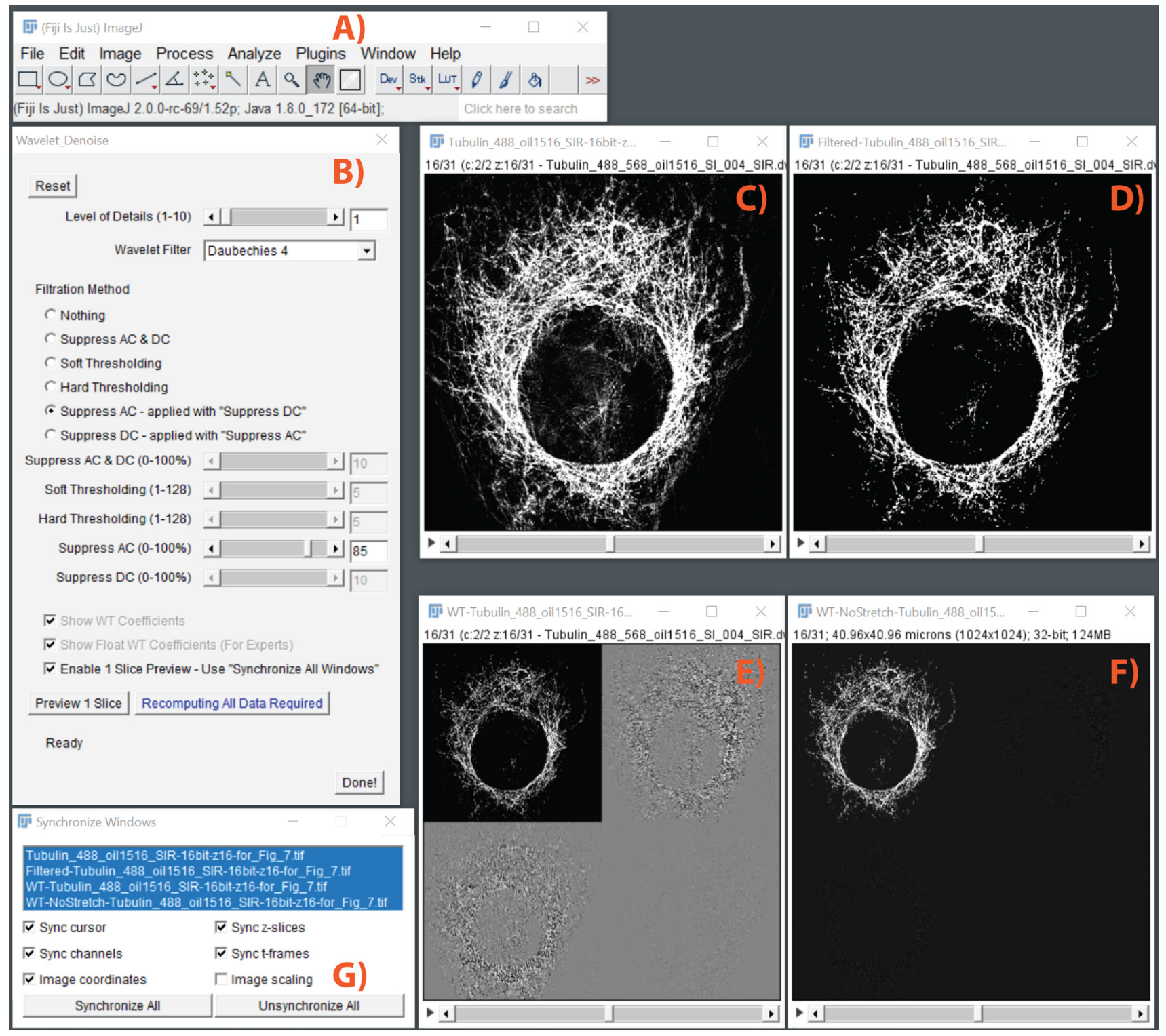

Fig. 7. Screenshot of Wavelet_Denoise plugin and images: A) Fiji window; B) Plugin dialog; C) Original data (tubulin network of a cell acquired by SIM); D) Filtered data; E) Wavelet decomposition; F) Wavelet decomposition in float precision; G) Synchronize Windows dialog, see also the text. 
Level of Details can be chosen in the first slider. This means that we influence the depth of analysis and the following synthesis, as shown in Fig. 4-5. A relatively large set of wavelet filters has been implemented (Haar, Daubechies 1-20, Symlets 2-20, Coiflets 1-5, Biorthogonal 1.1-6.8, Reverse Biorthogonal 1.1-6.8, Discrete Meyer) and can be selected in the corresponding pop-up menu (Fig. 7B). An interested reader can find visualization of some common scaling and wavelet functions using, e.g., this web address: https://vincentherrmann.github.io/blog/d3-test/.

Processing (denoising and filtration) methods in the wavelet domain are offered in the group of radio buttons. Nothing means no processing is applied. Selecting Suppress Approx. \& Detail. Coeffs. activates the corresponding slider (Suppress AC \& DC (0-100\%)) and removes chosen amount of ordered smallest coefficients, both approximation and detailed. User sets the level of suppression that equals the percentage of the coefficients to be removed (i.e., $0 \%$ means no values are suppressed, 100\% means all values are suppressed).

Denoise tools demonstrate the use of wavelet transform for image denoising. It is accomplished using thresholding (more specifically VisuShrink thresholding methods (Rangarajan et al., 2002)). The user defines a threshold by selecting the level of denoising (that equals Sigma value of the Universal threshold) in the dialog. It can also be selected to do either Soft Thresholding or Hard Thresholding.

Next two choices (Suppress Approx. Coeffs. (AC), Suppress Detail. Coeffs. (DC)) work together. Functionality is similar like in the option Suppress Approx. \& Detail. Coeffs, but both $A C$ and $D C$ are suppressed separately. Working together means that a user can, e.g., choose suppressing $10 \%$ of $A C$ and $50 \%$ of $D C$ and both suppressions are applied to the original data at once.

When any parameter in the dialog is changed, both wavelet coefficients (Fig. 7E) and the reconstructed, filtered data (Fig. 7D) are recomputed instantly. For standard images or stacks this is a good option, however, for large 3D stacks it may become time-consuming. For this purpose, we implemented Enable 1 Slice Preview option that gives possibility to set any parameter in the dialog without instant recomputation. After setting the filtration parameters, pressing the button Preview 1 Slice computes the forward DWT, filtration and backward DWT for the actual slice only. Here we recommend to use Synchronize All Windows option in the Synchronize Windows dialog to visualize the same layers in all image windows. In case Preview 1 Slice button is pressed, another button - Recomputing All Data Required becomes active. It provides the option to recompute the whole stack after the user is satisfied with the filtration results found with using Preview 1 Slice button.

By pressing Recomputing All Data Required or by unchecking Enable 1 Slice Preview option, the whole input image/stack is recomputed using the last parameters set.

Fig. 7E represents the wavelet domain of the input picture. It is visualized when Show WT Coefficients option is checked (Fig. 7B). The image of the domain does not contain true values, but it is stretched to intensity range between 0 and 255 and optimized for visualization in order to see effects of suppressing or denoising. If the user wishes to get a picture of true coefficients, e.g., for next processing in Matlab, it is also possible to check Show Float WT Coefficients (For Experts). Then another picture with values in float precision of DWT will be opened (Fig. 7F).

By pressing the button Reset, it is possible to return back to all default values of the main dialog together with the corresponding update of the images. This dialog also shows an actual status of the dialog or progress of computation expressed in percent in the bottom line.

\section{RESULTS}

\section{FILTERING SIM IMAGES OF TUBULIN NETWORK}

The first example shows filtration of images acquired by DeltaVision OMX V4 with SIM and widefield modality (WF), respectively. The image represents a tubulin network in U2OS cells (human bone osteosarcoma) (Fig. 8A) stained with Alexa Fluor 488 and visualized with excitation/emission 488/504-552 nm. For comparison and evaluation, we also show the sample of the same field of view acquired by WF of Deltavision OMX V4 microscope (Fig. 8B), with similar excitation/emission.

Images were captured using a PLAN APO 60x OIL objective with NA 1.42. The SIM acquisition was achieved with 3 grid rotations and 5 grid shifts, final data was reconstructed by SoftWoRx software package (GE Healthcare, USA). The size of the reconstructed SIM image matrix was $1024 \times 1024$ pixels, 16 bit and the pixel size was $40 \mathrm{~nm} \times 40 \mathrm{~nm}$. The size of acquired WF image was $512 \times 512$ pixels, 16 bit, the pixel size was $80 \mathrm{~nm} \times 80 \mathrm{~nm}$.

All figures in Fig. 8 are accompanied with insets showing zoomed in parts of the original picture, where artifacts in the reconstructed data occur. It can be seen that artifacts, mostly honeycombs, apparent in the inset 

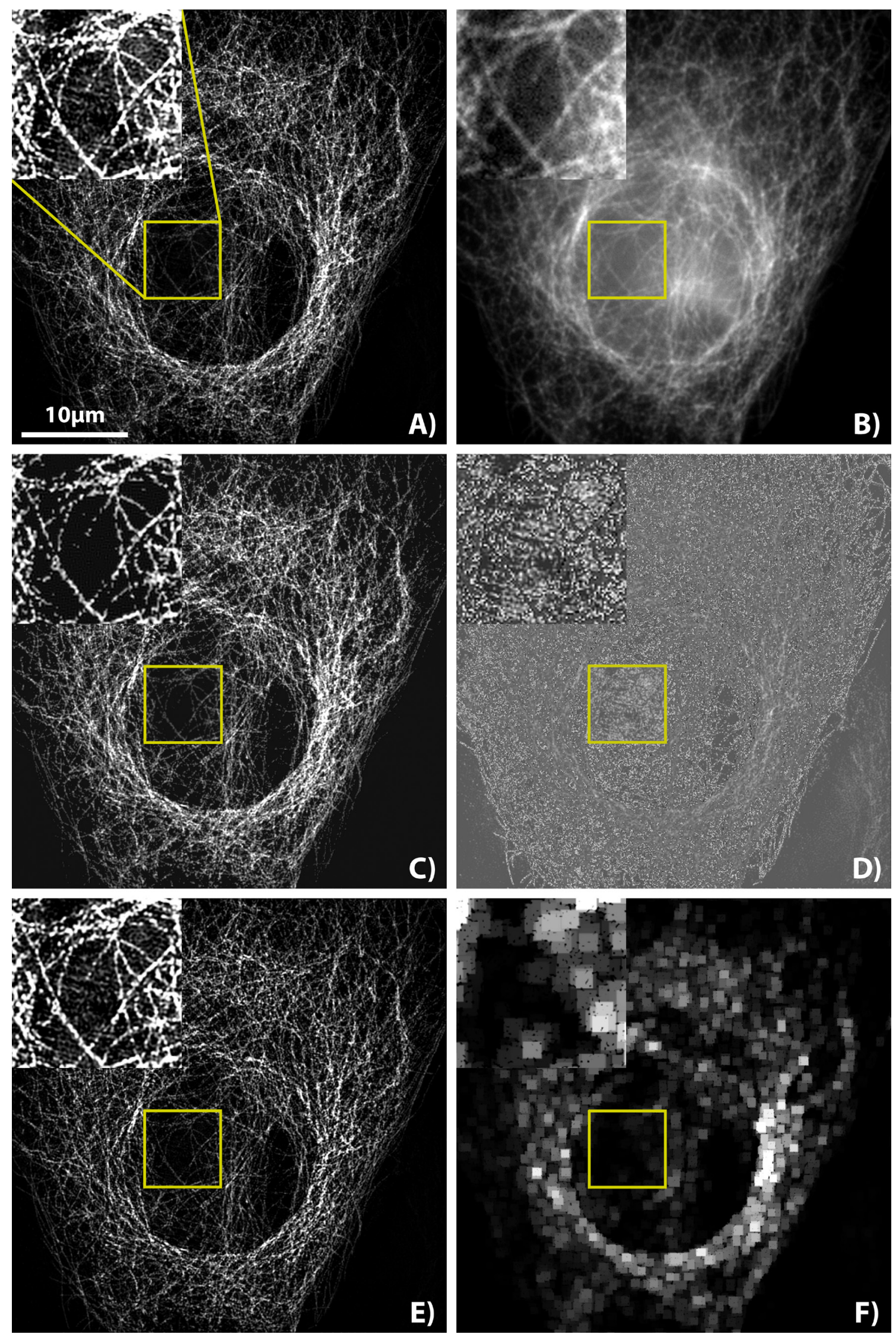

Fig. 8. Tubulin network in a U2OS cell: A) Original image - SIM; B) Original image - WF; C) SIM, DWT filtered, DB4, AC50; D) Differential image between original SIM and DWT filtered SIM (A vs. C); E) SIM, SB filtered, 10 pxl; F) Differential image between original and SB filtered SIM (A vs. E). See the text for details. 
in Fig. 8A (SIM) are not present in the inset in Fig. 8B (WF). It should be noted that all demonstrated images, including the insets, have contrast linearly increased for optimal visualization.

Fig. 8C depicts the data after applying DWT. Here, we chose Daubechies 4 (DB4) as a relatively easy wavelet and we restricted amount of approximation coefficients simply to $50 \%$ (AC50). The result is visualized best in the inset where practically all background artifacts disappeared and signal is mostly preserved. Fig. 8D shows a differential image between the original data (Fig. 8A) and the filtered data (Fig. 8C). Although parts of the signal were filtered out, we can observe that brightest structures visible in the difference image (Fig. 8D) are removed artifacts and/or background, which was desired.

In this example we also compared the wavelet filtration with a technique Subtract Background (https://imagejdocu.tudor.lu/gui/process/subtract background) that is used in Fiji for removing undesired background, i.e. for similar purposes like DWT filtration was used in this article. Subtract Background is good for removing smooth continuous background, e.g., from images of gels or microscopic images. We show it here for comparison, since it is an established and good background removing technique in ImageJ/Fiji.

Fig. 8E shows the original data after Subtract Background (SB) filtration with the Rolling ball radius 10 pixels. This parameter is set to be at least as large as the radius of the largest object in the image that is not part of the background. After linearly increasing contrast in
Fig. 8E we can observe that for this type of background the method does not work sufficiently. Overall intensity of the picture is lowered, but - as seen in the inset - artifacts are mostly preserved. It is also depicted in Fig. $8 \mathrm{~F}$, which is a differential image between Fig. 8A and Fig. 8E. One can notice that this kind of filtration influenced especially brightest parts of the signal, but artifacts were filtered out less efficiently when compared with DWT filtration (Fig. 8C, D).

\section{PORES \\ FILTERING SIM IMAGES OF NUCLEAR}

In the next example we show the effect of various strengths of DWT filtration with respect to an image quality criterion that compares a filtered picture of a structure with another picture of the same structure acquired by different imaging modality. For this purpose, we apply NanoJ-SQUIRREL plugin (Culley et al., 2018) as an efficient and modern tool for evaluating quality of multimodal data. This plugin uses Resolution Scaled Pearson coefficient (RSP), which is standard Pearson correlation coefficient adapted to data acquired by various imaging modalities with different point spread functions (PSF) and data with different intensities, pixel sizes and pixel numbers.

In a nutshell, the plugin evaluates super-resolution images with a reference image of the same structure (typically a widefield, TIRF or confocal image). We apply the RSP to compare super-resolution STED images of cells with nuclear pores with respect to super-resolution SIM images of the same cells with and without graduated strength of DWT filtration.

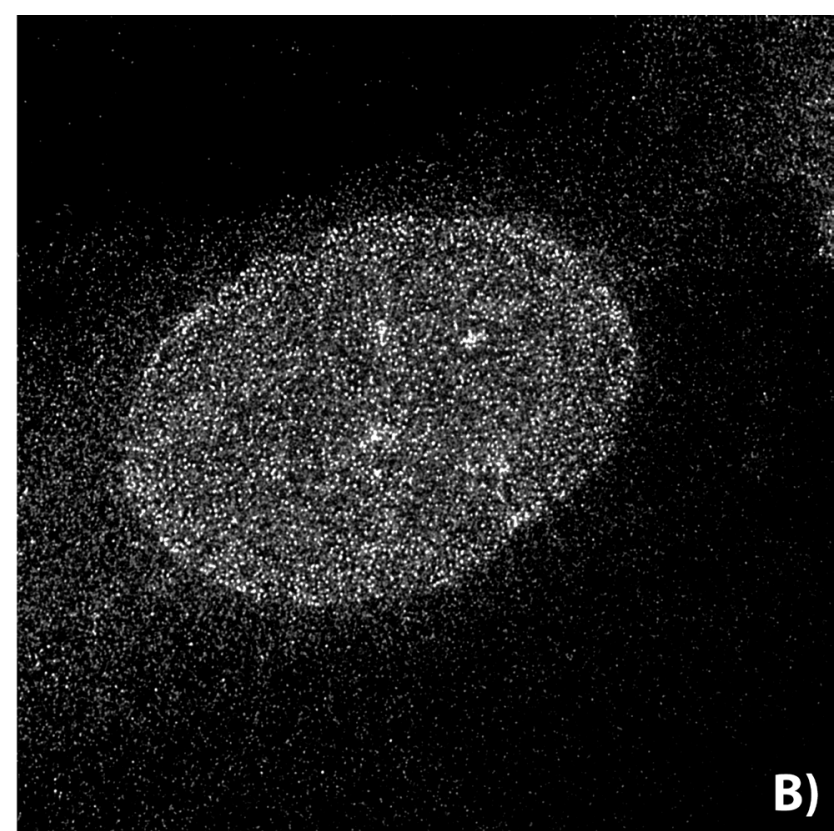

Fig. 9. U2OS cell with nuclear pores, acquired by SIM (A) and STED (B). 
Nuclear pores of U2OS cells were stained with Alexa Fluor 555. We acquired images of four cells with the pores, firstly, by Deltavision OMX V4 microscope (PLAN APO 60x/1.42 OIL objective) in SIM mode, with excitation/emission 568/591-627 nm and, secondly, by Leica TCS SP8 STED 3X microscope (HC PL APO 100x/1.4 OIL STED objective, $660 \mathrm{~nm}$ CW STED depletion laser) in STED mode, with excitation/emission 553/563-630 nm. See an example of images of one such a cell in Fig. 9.

The size of SIM image matrices, reconstructed by SoftWoRx software package (GE Healthcare, USA), were $1024 \times 1024$ pixels, 16 bit and the pixel size was $40 \mathrm{~nm} \times 40 \mathrm{~nm}$. The size of acquired STED images was $2048 \times 2048$ pixels, 8 bit, the pixel size was $20.18 \mathrm{~nm} \times 20.18 \mathrm{~nm}$. The STED data were further deconvolved by Huygens Professional software package

\section{Cell 1}

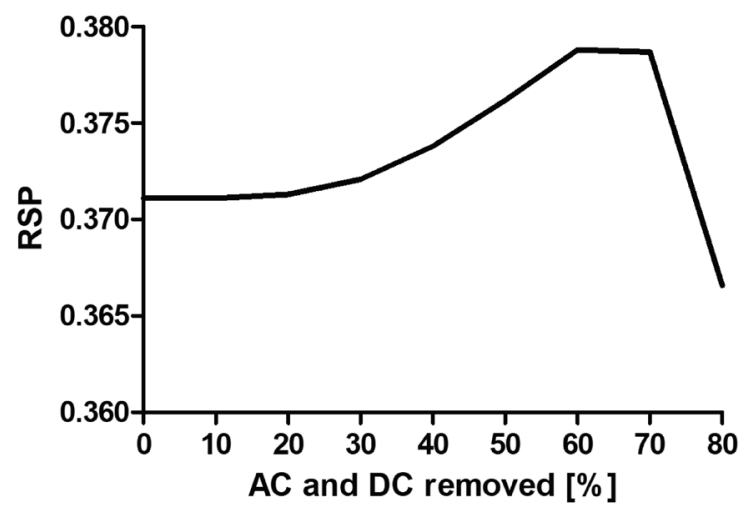

Cell 3

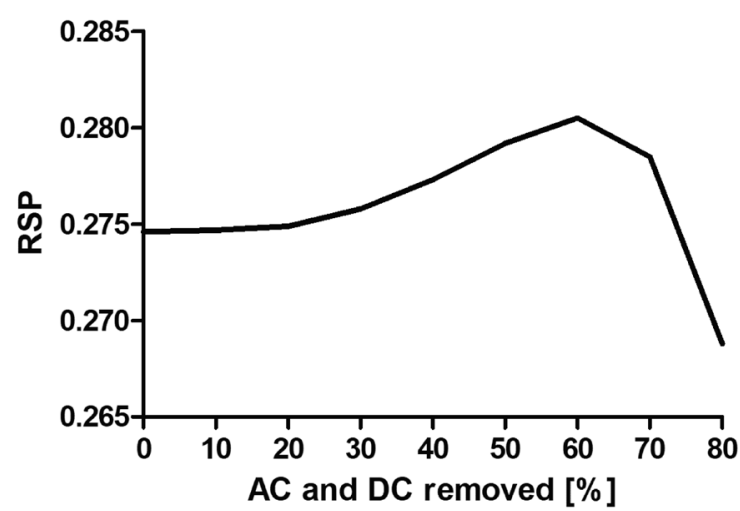

(https://svi.nl/HomePage) to improve their quality and converted to 16 bit depth.

We processed the data by NanoJ-SQUIRREL plugin, an interested reader can also visit https://henriqueslab.github.io/resources/NanoJ-SQUIRREL/. The plugin requires choosing Super-Resolution Reconstruction $(s)$ - we used STED images here as data with high microscopic resolution - and Reference Image - we applied SIM data, both original and filtered with graduated strength of filtration. We applied values of DWT filtration increased by steps of $10 \%$ in both parameters, starting from $A C 0 \%, D C 0 \%$ and ending with $A C 80 \%, D C$ $80 \%$. The results are collected in Fig 10. It appears optimal, from the point of view of the used criterion and for such kind of data, to apply both $A C$ and $D C$ parameters in the interval around $40 \%-70 \%$ to get maximum similarity, evaluated by RSP, between filtered SIM and STED data.

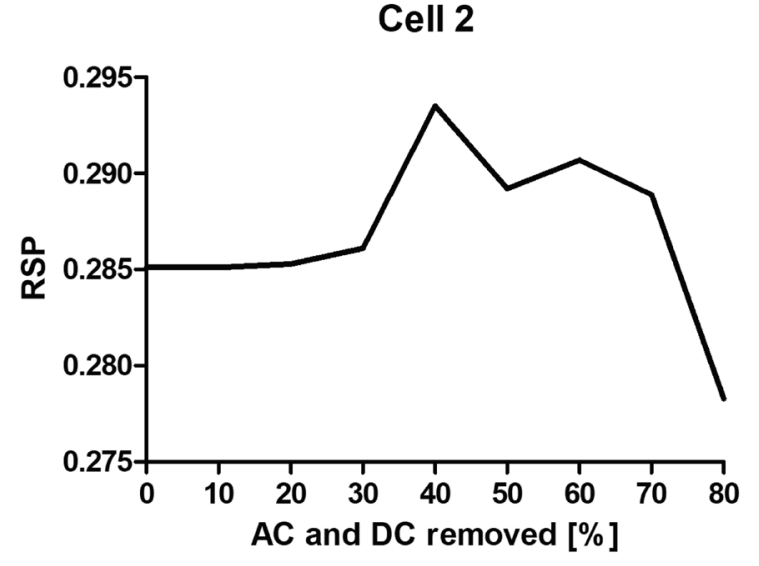

\section{Cell 4}

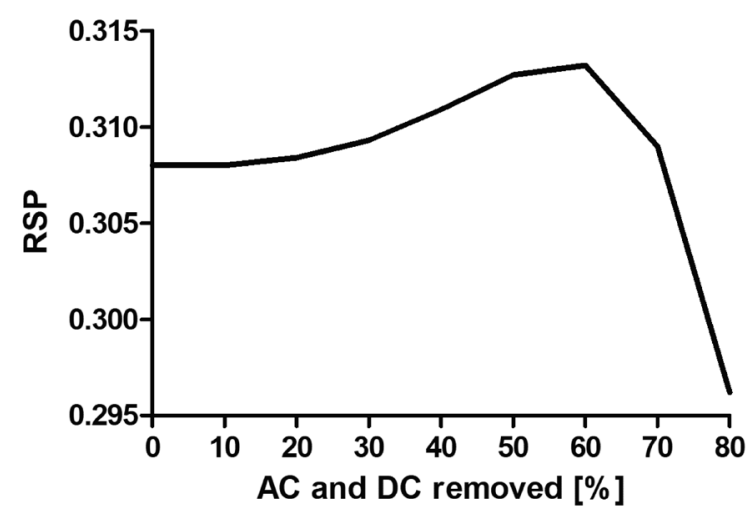

Fig. 10. Charts with Resolution Scaled Pearson coefficient (RSP) computed for images of four cells with nuclear pores and for various strengths of DWT filtration (in percents of $A C$ and DC removed). 

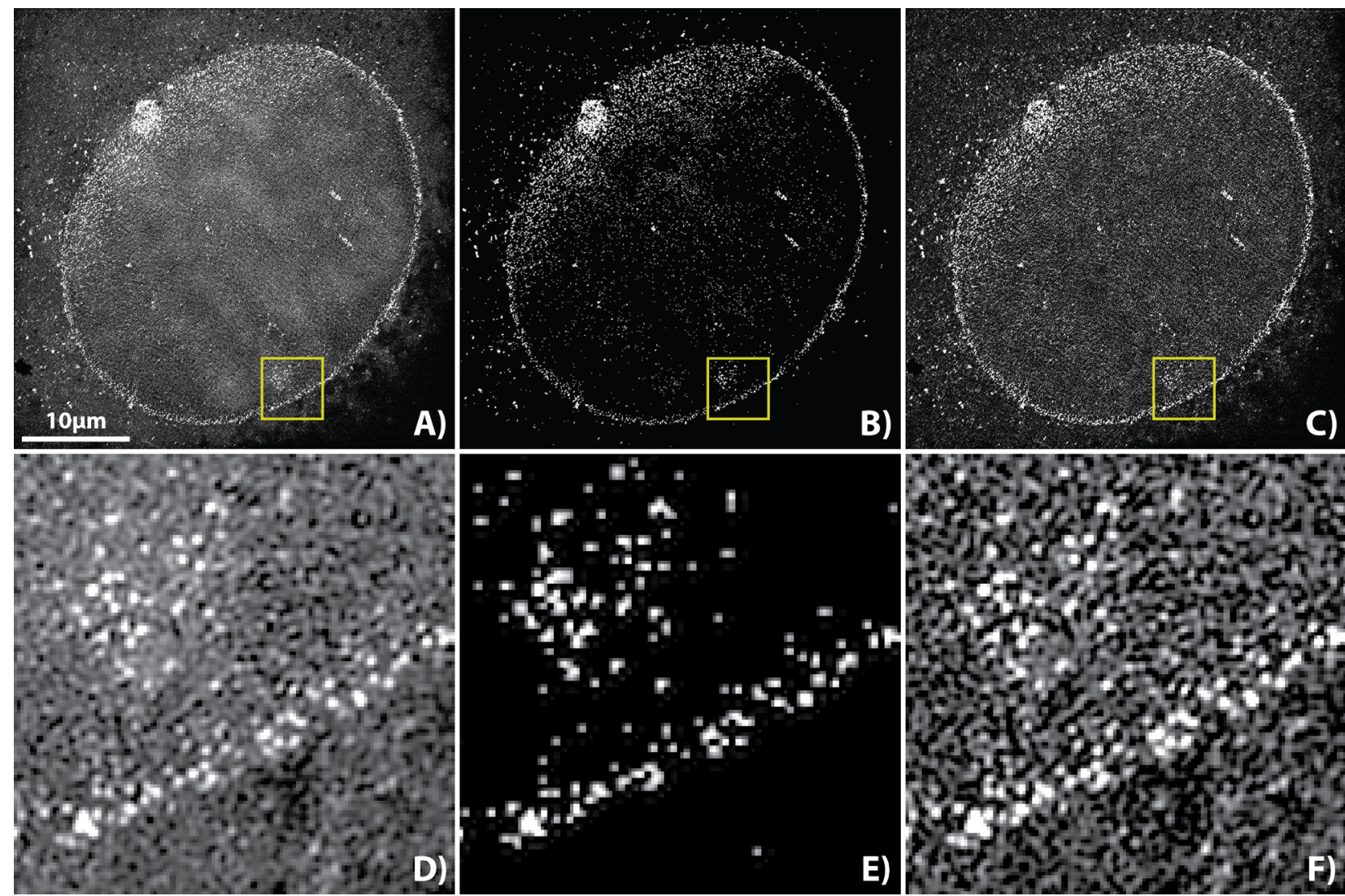

Fig. 11. U2OS cell with nuclear pores showing strong artifacts with yellow square areas in the same position, SIM: A) Original image; B) DWT filtered, DB4, AC95; C) SB filtered, $10 \mathrm{pxl}$; D) Zoomed in yellow square area in A; E) Zoomed in area in $B ; F$ ) Zoomed in area in $C$.

\section{FILTERING OUT STRONG ARTIFACTS}

In Fig. 11 we exemplify the effect of DWT filtration applied to a picture of a U2OS cell with stained nuclear pores introducing strong reconstruction (honeycomb) artifacts. We compare original data (Fig. 11A) with a zoomed in part (Fig. 11D) with highly filtered DWT data $(A C=95 \%$; Fig. $11 \mathrm{~B}$ and $\mathrm{E}$ ) and with data after applying

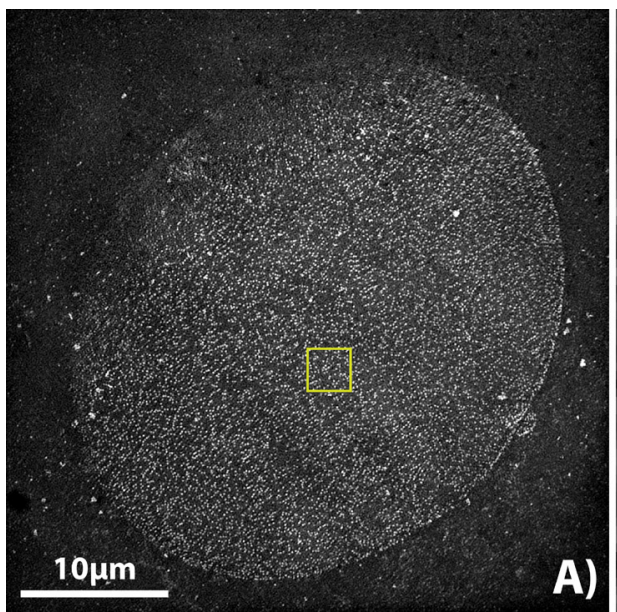

SB filtration (Fig. 11C and F).

With the DWT filtration, we can see the mostly preserved bright pores and efficiently removed background artifacts. In case of SB filtration background artifacts were preserved, their intensity was only lowered. It confirms better suitability of the DWT filtration for such kind of image enhancement than SB filtration.

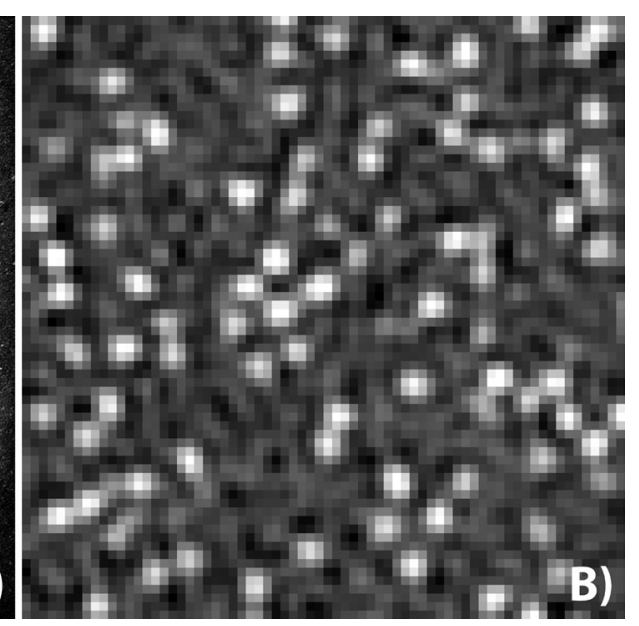

Fig. 12. A) An image of U2OS cell with a yellow ROI, B) zoomed in ROI from A) showing nuclear pores (white) and structures in the background. 


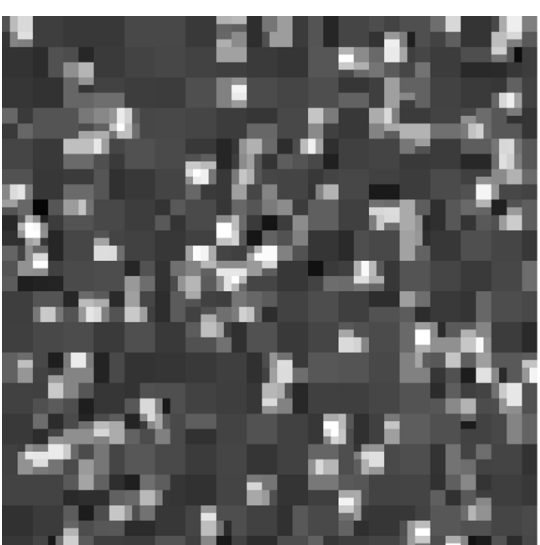

A) Suppress AC \& DC 90\%, Daubechies 1

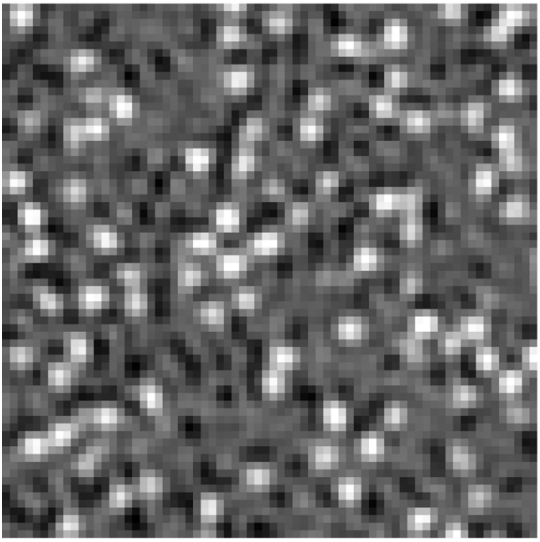

D) Daubechies 4, Suppress AC $\mathbf{5 0 \%}$

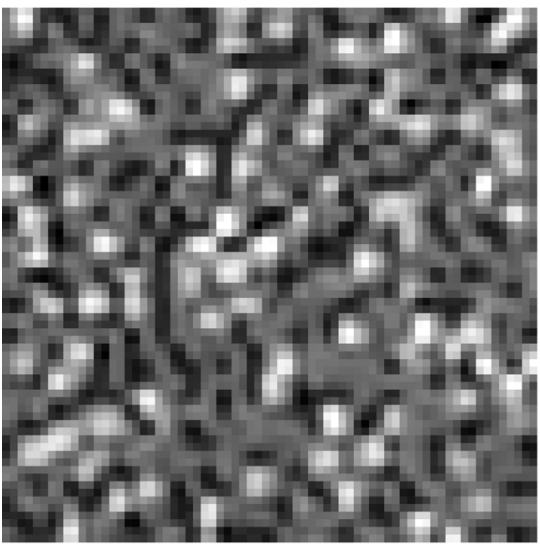

G) Daubechies 4, Suppress AC 90\%

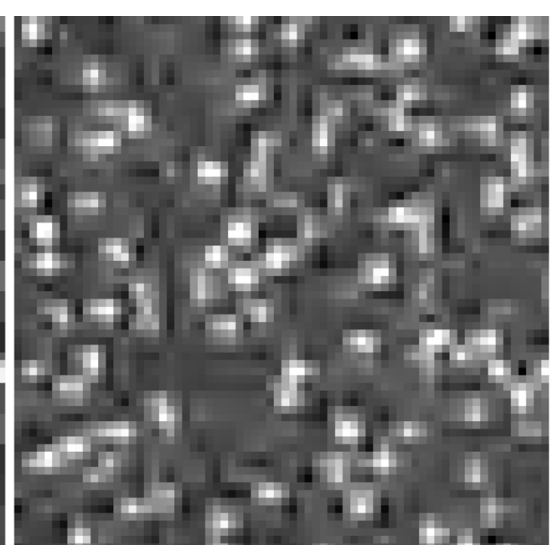

B) Suppress AC \& DC 90\%, Daubechies 2

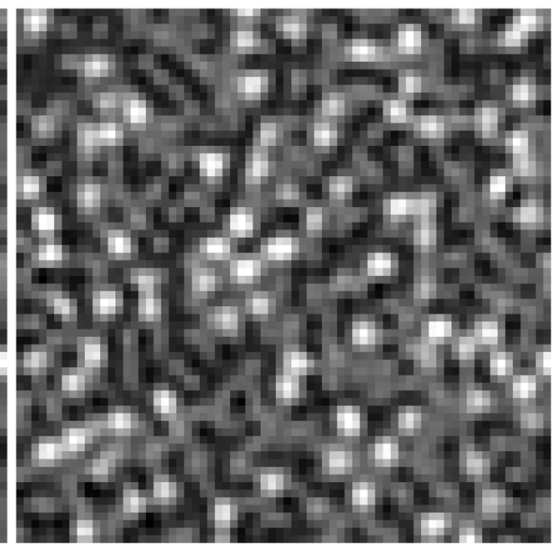

E) Daubechies 4, Suppress AC 70\%

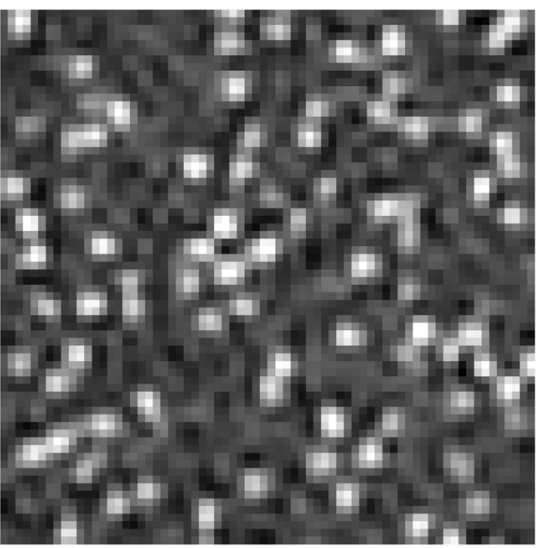

H) Daubechies 4, Soft Thresholding $90 \%$

(corresponding to value 115 from

maximum 128 in the plugin dialog)

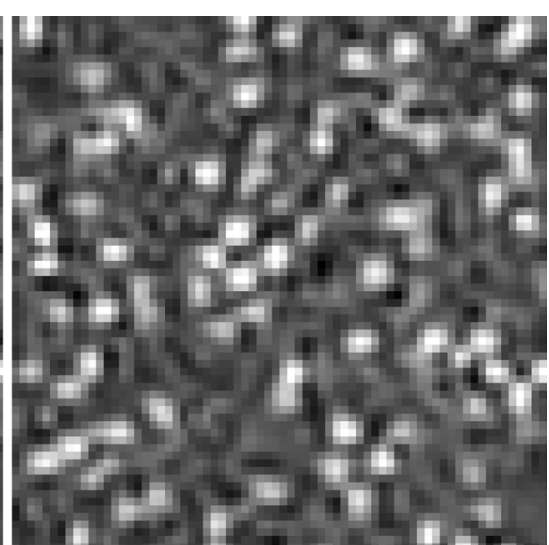

C) Suppress AC \& DC 90\%, Daubechies 10

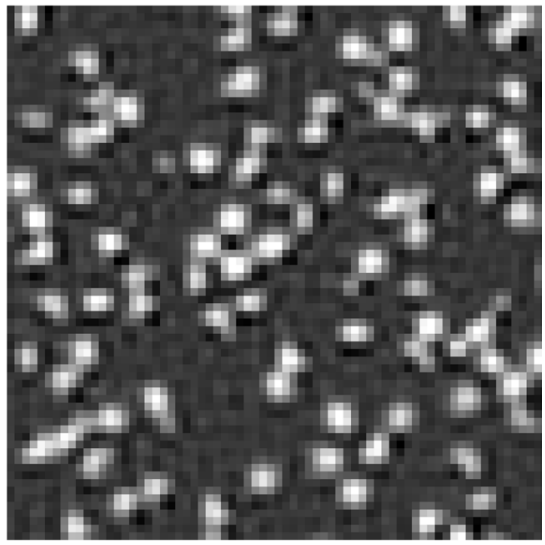

F) Daubechies 4, Suppress AC $\mathbf{9 0 \%}$

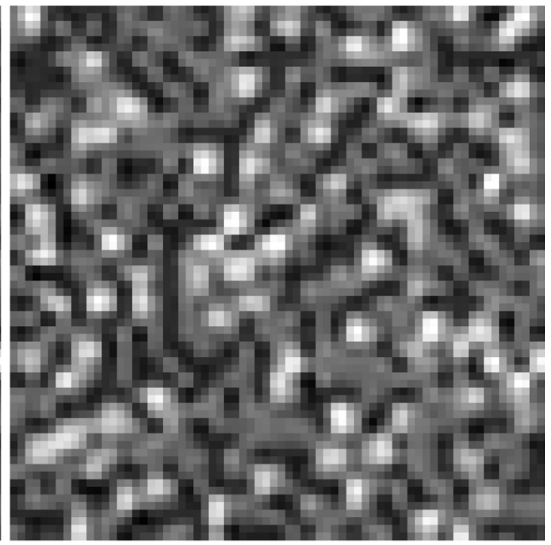

I) Daubechies 4, Hard Thresholding 90\%

(corresponding to value 115 from

maximum 128 in the plugin dialog)

Fig. 13. Image subsets from the same position of ROI in Fig. 12B showing its filtration by various wavelet filters, various filter powers and various filtration methods: First row $(A-C)$ - the same method applied to the data (Suppress $A C \& D C$, Level of Details 2), the same power of the method applied (90\%), but various wavelet filters (Daubechies 1, 2, 10) demonstrating that high order wavelet filters support better compression of data. Although 90\% of wavelet coefficients was removed from reconstructed data, the picture C), with using Daubechies 10, preserves image details best. Second row (D-F) - the same method applied to the data (Suppress AC, Level of Details 1), the same wavelet filter (Daubechies 4), but various strengths of the filter $(50 \%, 70 \%, 90 \%)$, exemplifying the power of filtration of image background. For $90 \%$ practically only nuclear pores were preserved. Third row (G-I) - the same wavelet filter (Daubechies 4, Level of Details 1), approximately the same strength of the filter (90\%), but various methods applied to the data (Suppress AC, Soft Thresholding, Hard Thresholding) depicting that Suppress AC and Hard Thresholding provide similar results, since both methods remove high portion of approximation coefficients from reconstructions. On the other hand, Soft Thresholding removes mainly detailed coefficients, i.e. noise, thus the picture is smoothed, but otherwise it is similar to the original image (Fig. 12B). 


\section{USING VARIOUS PLUGIN SETTINGS TO FILTER AN IMAGE}

In the last paragraph, we would like to demonstrate the use of different settings of the developed plugin to process an area of the microscopic image, see Fig. 12. The results of applying various settings of wavelet filtration to the area in Fig. 12B are shown in Fig. 13. The given figure nicely demonstrates the various possibilities and effects of such filtering and denoising.

\section{DISCUSSION}

We have introduced Wavelet_Denoise plugin for Fiji software package devoted to filtration and denoising of images and shown its main features. The plugin can be applied to various kind of pictures. We have also demonstrated its successful application to images acquired by structured illumination microscopy. We compared quality of filtered data with images of the same structure acquired by another super-resolution microscopic technique - STED - as well. We propose the range of $A C$ and $D C$ filtration parameters, i.e. $40 \%-70 \%$, for optimal filtration of data from the point of view of Resolution Scaled Pearson (RSP) criterion as suggested in Culley et al., 2018.

Plugin Wavelet_Denoise can be installed in Fiji using Help $\rightarrow$ Update... menu command. Afterwards, through choosing LMCF-IMG update site and Fiji restarting the plugin is accessible in Plugins $\rightarrow L M C F$ $I M G$ menu item. More about installing Fiji plugins can be found online (https://imagej.net/Following an update site).

Additional information about our plugin, manuals for processing data of various sizes and example images can be found in the wiki page (https://imagej.net/Wavelet_Denoise).

We conclude that by the development of Wavelet_Denoise plugin we provided the community with a free and efficient tool for processing not only microscopic images.

\section{ACKNOWLEDGEMENTS}

We greatly acknowledge support of Ministry of Education, Youth and Sports of the Czech Republic using the following funds: "National Infrastructure for Biological and Medical Imaging" (LM2018129), "Modernization and support of research activities of the national infrastructure for biological and medical imaging CzechBioImaging" (CZ.02.1.01/0.0/0.0/16_013/0001775), "Centre of Model Organisms" (CZ.2.16/3.1.00/21547) and "Biomodels for health - Centre of Model Organisms" (LO1419).

\section{REFERENCES}

Antonini M, Barlaud M, Mathieu P, Daubechies I (1992). Image coding using wavelet transform. IEEE T Image Process 1(2):205-20.

Ball G, Demmerle J, Kaufmann R, Davis I, Dobbie IM, Schermelleh L (2015). SIMcheck: a toolbox for successful super-resolution structured illumination microscopy. Sci Rep 5, 15915, 12 p.

Born M, Wolf E (1997). Principles of Optics. Cambridge University Press, 808 p.

Culley S, Albrecht D, Jacobs C, Pereira PM, Leterrier C, Mercer J, Henriques R (2018). Quantitative mapping and minimization of super-resolution optical imaging artifacts. Nat Methods 15:263-6.

Chui C (1992). An introduction to wavelets. 1st ed. Boston: Academic Press, 264 p.

Demmerle J, Innocent C, North AJ, Ball G, Müller M, Miron E, Matsuda A, Dobbie IM, Markaki Y, Schermelleh L (2017). Strategic and practical guidelines for successful structured illumination microscopy. Nat Protoc 12(5):988-1010.

Gonzales RC, Woods RE (2008). Digital image processing. 3rd ed. Pearson Prentice Hall, 954 p.

Gustafsson MGL (2000). Surpassing the lateral resolution limit by a factor of two using structured illumination microscopy. J Microsc 198(2):82-7.

Gustafsson MGL, Shao L, Carlton PM, Wang CJR, Golubovskaya IN, Cande WZ, Agard DA, Sedat JW (2008). Three-dimensional resolution doubling in wide-field fluorescence microscopy by structured illumination. Biophys J 94(12):4957-70.

Kř́žžek P, Lukeš T, Ovesný M, Fliegel K, Hagen GM (2016). SIMToolbox: a MATLAB toolbox for structured illumination fluorescence microscopy. Bioinformatics 32(2):318-20.

Luisier F, Blu T (2008). SURE-LET Multichannel Image Denoising: Interscale Orthonormal Wavelet Thresholding. IEEE T Image Process 17(4):482-92.

Luisier F, Blu T, Unser M (2007). A New SURE Approach to Image Denoising: Interscale Orthonormal Wavelet Thresholding. IEEE T Image Process 16(3):593-606.

Rangarajan R, Venkataramanan R, Shan S (2002). Image Denoising Using Wavelets. Proceedings of Wavelets \& Time Frequency, University of Michigan, $13 \mathrm{p}$.

Righolt CH, Slotman JA, Young IT, Mai S, van Vliet LJ, Stallinga $S$ (2013). Image filtering in structured illumination microscopy using the Lukosz bound. Opt Express 21:24431-51. 
Roselló EG, Dacosta JG, Lado MJ, Méndez AJ, Sampedro J, Cota MP (2014). Visual Wavelet-Lab: An objectoriented library and a GUI application for the study of the wavelet transform. Comput Appl Eng Educ 22(1):23-32.

Royon A, Converset N (2017). Quality Control of Fluorescence Imaging Systems: A new tool for performance assessment and monitoring. Optical Measurement and Testing, 12(2):22-5.

Sage D, Unser M (2003). Teaching Image-Processing Programming in Java. IEEE Signal Process Mag 20(6):4352.
Sečkář P (2016). E-learning application for wavelet transform and its optimized GPU implementation, Masaryk University, Brno, 55p.

Strang G, Nguyen T (1997). Wavelets and filter banks. Wellesley: Wellesley-Cambridge Press, 520 p.

Unser M, Blu T (2000). Fractional Splines and Wavelets. SIAM Rev 42(1):43-67. 